\title{
5 Research Square

\section{Numerical Study of the Effects of an Obstacle and its Position in a Curved Channel in a Lock-Exchange Flow}

Javad Mohammadi

University of Sistan and Baluchestan

Bahareh Pirzadeh ( $\boldsymbol{D}$ b_pirzadeh@eng.usb.ac.ir)

University of Sistan and Baluchestan https://orcid.org/0000-0002-5728-3481

Gholamreza Azizyan

University of Sistan and Baluchestan

Azam Abdollahi

University of Sistan and Baluchestan

\section{Research Article}

Keywords: Gravity Current, Curved Channel, obstacle , Lock-exchange, OpenFOAM

Posted Date: July 20th, 2021

DOI: https://doi.org/10.21203/rs.3.rs-708438/v2

License: @ (i) This work is licensed under a Creative Commons Attribution 4.0 International License.

Read Full License 


\title{
Numerical Study of the Effects of an Obstacle and its Position in a Curved Channel in a Lock-Exchange Flow
}

\author{
Javad Mohammadi ${ }^{1}$, Bahareh Pirzadeh ${ }^{2 *}$, Gholamreza Azizyan $^{3}$, Azam Abdollahi $^{4}$ \\ ${ }^{1}$ Master student, Civil Engineering Department, University of Sistan and Baluchestan, Zahedan, Iran; \\ (Email: javad.mohamadi5688@gmail.com) \\ ${ }^{2}$ Assistant Professor, Civil Engineering Department, University of Sistan and Baluchestan, Zahedan, \\ Iran; *Corresponding author (Email: b_pirzadeh@eng.usb.ac.ir)
}

${ }^{3}$ Associate Professor, Civil Engineering Department, University of Sistan and Baluchestan, Zahedan, Iran; (Email: g.azizyan@eng.usb.ac.ir)

${ }^{4}$ Ph.D., Civil Engineering Department, University of Sistan and Baluchestan, Zahedan, Iran; (Email: azam.abdollahi@pgs.usb.ac.ir)

\begin{abstract}
Researchers have recently shown interest in complex flow patterns, especially the effects of secondary flows, in a curved channel. This paper studied the obstacle effects in a gravity current in a channel with a symmetrical $120^{\circ}$ bend using the OpenFoam toolbox and the Realizable k$\varepsilon$ turbulence model for simulation. The models studied included no-obstacle curved channel, curved channel with obstacle in $30^{\circ}$ position, curved channel with obstacle in $60^{\circ}$ position and curved channel with obstacle in $60^{\circ}$ position with increased radius. Results showed that the obstacle directed the concentration towards the banks with its maximum value tending from the outer to the inner bank, especially in the tail. Although the post-obstacle head height did not change, that of the tail did (fell); the tail longitudinal velocity was maximized near the channel bed in areas far from the obstacle, and in the outer bank in areas near it. The secondary flow was so reduced that its lowest and most different pattern was observed around the obstacle.
\end{abstract}


In displacing the latter, if the front was at a certain distance from it, the secondary flow did not change much, but if it was at the channel end, the post-obstacle secondary flow increased as the obstacle neared the lock.

KEYWORDS: Gravity Current -Curved Channel- obstacle - Lock-exchange - OpenFOAM Secondary Flow 


\section{INTRODUCTION}

Gravity currents are two-phase flows formed due to the density difference between two fluids because of engineering reasons, natural phenomena (avalanche, sea breeze, sandstorm, etc.) or human interference (discharging pollutants into rivers and dams' reservoirs, tunnel fires, etc.) (Simpson, 1999; Meiburg et al., 2015). Such factors as temperature variations, chemical compounds and solid particles can cause density differences, and since solid particles are difficult to simulate, use can be made of high-density salt water solutions for related studies (Ooi et al., 2007; Paik et al., 2009). Ungarish (2009) describes formation conditions of the gravity current $\left(\rho_{\mathrm{c}}>\rho_{\mathrm{a}}\right)$ under Lock-exchange topics two dimensionally at position zero $\left(x_{0}\right)$ (Fig. 1). According to Fig.1, the interface under consideration in the present configuration has two components. The first is a vertical front (also referred to as nose) and the second, and major, part of the interface is the inclined, sometimes piecewise inclined-horizontal, surface (Ungarish, 2009).

With the gate removal at $x_{0}$, denser current begins to have a sharp front somewhere near the floor wall, forms along the channel base and becomes more along the flow (Hacker et al., 1996).

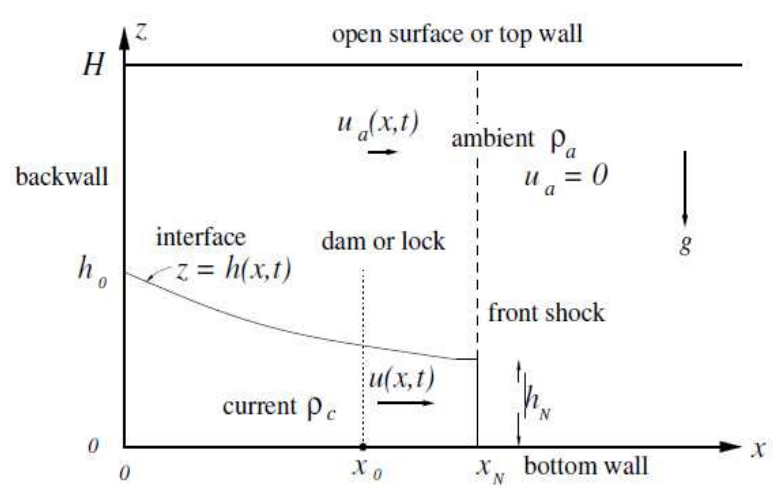

Fig. 1. Schematic description of the gravity current configurations for a bottom current of heavy fluid released from behind a lock (Ungarish, 2009)

Hacker et al. (1996) was the first to do gravity-current lab studies in high-Reynolds Lockexchange topics where the two fluids' density difference was $12 \%$, the denser fluid's volume 
was $0.0328 \mathrm{~m} 3$, and Reynolds number was 19700. Paik et al. (2009), Ooi et al. (2007) and Mahdinia et al. (2012) are among many researchers who based their works on this experiment; the present study has used the configuration of the Hacker test. Mahdinia et al. (2012), who simulated, for the first time, the gravity-current in bend under Lock-exchange, believed that such currents could occur in rivers, at the entrance of reservoirs or in any other natural environment. Following the latter work, the present study has used an obstacle in a curved channel to study gravity-currents' hydraulic properties.

Examples of comprehensive studies on gravity currents in direct channels under Lockexchange conditions include those of Firoozabadi et al. (2009) who simulated the velocity/density of channels' inflows, Lam et al. (2018) and Ottolenghi et al. (2016) who simulated lock dimensions variations and density difference of two fluids, Zhao et al. (2018), Lombardi et al. (2015) and Ottolenghi et al. (2016) who simulated the channel gradient flow hydrodynamics, and so on; Longo's (2015) too is among limited studies on the effects of the cross-section type on the flow. The modified RANS model introduced by Paik et al. (2009) observed near-wall flows well. Ooi's (2007) study results showed that it is better, in numerical studies, to use the no-slip boundary condition for walls so that density lines could have better validations. Among those who studied obstacle and roughness effects on the gravity current in direct channels, Tokyay et al. (2012) investigated the roughness effects on the channel bed wall shear stress in one study, and the flow regime due to the obstacle height variations in another (Tokyay and Constantinescu, 2015).

Lock-exchange experiments were used to examine the temporal entrainment properties and mixing processes of sediment-laden turbidity currents interacting with a rectangular obstacle. During the slumping process, currents of varying densities were studied over smooth and rough substrates (Wilson et al. 2017). 
The entrainment and mixing dynamics of a gravity current produced by a lock-exchange mechanism were investigated experimentally by simultaneously measuring velocity and density fields. Near the front of the gravity present, the values of entrainment coefficient were found to be the largest (Balasubramanian and Zhong, 2018).

To find out how three-dimensional canopy geometry affects the front propagation of an incoming gravity current under a given initial forcing, large-eddy simulations were conducted by Zhou and Venayagamoorthy, 2020. It was shown that when applied to buoyancy-driven flows, the traditional geometrical parameter of submerged canopies in constant-density flows is misleading.

In a general geometry-based grouping, Brice et al. (1974) divides meanders into simple symmetric, simple asymmetries, symmetric combination and asymmetric combination. Based on his studies and morphological analyses, Magdaleno et al. (2011) believes that rivers naturally tend to have a symmetrical form.

Bend-geometry open-channel fellow studies are many; Abad and Garcia (2009a) measurements in an asymmetric lab channel show that the maximum velocity occurs at the flow surface in the inner bank of the bend, and at the channel bed in the outer bank of the bend; in the channel width, the highest velocity difference is observed at the bend vertex. Next in his research, Abad added the erodible bed to the test conditions and studied movements of sediment particles (Abad and Garcia, 2009b). In a numerical study, Farshi et al (2018) presented relationships for secondary flows in $120^{\circ}$ symmetric bends in a flood channel and showed, by the flow pattern, that the secondary current increased after the bend vertex and reached its maximum near the outer bank; the pattern was, then, repeated from this vertex to the next. 
Flow patterns in many studies (Kassem et al., 2004; Islam and Imran., 2008; Ezz et al., 2013 and Sumner et al., 2014) on submarine density currents, formed like a bend under Coriolis force effects of sinusoidal currents, have similarities and differences compared to those in open channel bends.

Since Mahdinia et al. (2012) is the only one who studied gravity currents in curved channels under Lock-exchange conditions and showed that mid-flow (somewhere between head and tail) has features similar to submarine density currents, his study is of particular importance to the present work. For instance, the flow, in both cases, increases in the outer bank and secondary currents occur at the bend vertex near the canal bed. In his study, while there is almost no secondary flow at the head, the one near the bed has a direction toward the outer bank. Near the tail, the pattern is different due, maybe, to the reduced density/velocity that has no definite direction across the channel width; the highest longitudinal velocity too occurs in the bend's outer bank. He, then, studied the effects of the bend radius and showed that its decrease increased the secondary flow because the centrifugal force and bend radius are inversely related.

According to the literature, as no studies have been done so far on the obstacle effects in curved channels under gravity currents, this paper has used an obstacle in a $120^{\circ}$ curved channel to study the hydraulic features (e.g., gravity and velocity) of the flow and location effects of the obstacle by some numerical modellings.

\section{NUMERICAL METHODS}

For modeling, this research has used OpenFOAM, which is an open-source and uses the $\mathrm{C}++$ language to solve CFD problems, and the twoliqiudMixingFoam solver, which is used in Lock exchange cases and is defined for 2-phase currents where both phases are incompressible and have different densities. 
For velocity and coupling it with pressure, use has been made of the noSlip boundary condition [Ooi et al., 2007] and the PIMPLE algorithm, respectively, and the boundary condition for pressure at all boundaries is the fixedFluxPressure. Wall functions are used for $\vartheta_{t}$, $\mathrm{k}$, and $\varepsilon$.

\section{GOVERNING EQUATIONS AND TURBULENCE MODELING}

The twoLiquidMixingFoam is an open-source solver form miscible and incompressible fluid. The solver finds the volume fraction $\alpha_{0}$ and $\alpha_{1}=1-\alpha_{0}$, and then the velocity $U$ by alpha diffusion equation, the continuity, and the momentum equations (Xie and Chu, 2019):

$$
\begin{aligned}
& \frac{\partial \alpha_{0}}{\partial t}+\nabla \cdot\left(U \alpha_{0}\right)=\nabla \cdot\left(\left(D_{a b}+\frac{\vartheta_{t}}{S_{C}}\right) \nabla \alpha_{0}\right) \\
& \nabla \cdot U=0 \\
& \frac{\partial \rho U}{\partial t}+\nabla \cdot \rho U U=-\nabla \cdot p_{r g h}+\nabla \cdot(\rho \tau)-g h h \nabla \rho
\end{aligned}
$$

Where $D_{a b}$ is the molecular diffusivity and $S_{c}$ is the turbulent Schmidth number. More details of this solver and its equations in numerical simulation of related problems can be found in (Zhang et al, 2016 and Xie and Chu, 2019).

For the Lock-exchange, values of the Froude and Reynolds numbers $\left(F r=u_{f} / u_{b}\right.$ and $R e=$ $u_{f} h / 2 v$ equal to 0.45 and 19700 , respectively) are those in the second test of Hacker et al. (1996) which is the validation model of the present and Mahdinia et al. (2012) papers. In these relations, $h, u_{f}$ and $v$ are the lock height at zero moment, current head velocity in direct channel, and dense fluid viscosity, respectively, $u_{b}$ is the buoyancy velocity equal to $\sqrt{g^{\prime} h}$ where $g^{\prime}$ is the reduced gravitational acceleration equal to $g\left(\rho_{\max }-\rho_{\min }\right) / \rho_{\min }$ where $g$ is the earth gravitational acceleration, $\rho_{\max }$ is the fluid density inside the lock, $\rho_{\min }$ is that in the ambient fluids and time $t_{0}$ equals $h / u_{b}$. 
From among RANS models, this study has selected the realizable $k-\varepsilon$ turbulence model wherein the kinetic energy (k) and energy dissipation ( $(\varepsilon)$ are as follows (Shaheed et al., 2019):

$$
\begin{gathered}
\frac{\partial k}{\partial t}+\frac{\partial k u_{i}}{\partial x_{i}}=\frac{\partial}{\partial x_{i}}\left(D k_{e f f} \frac{\partial k}{\partial x_{i}}\right)+G_{k}-\varepsilon \\
\frac{\partial \varepsilon}{\partial t}+\frac{\partial \varepsilon u_{i}}{\partial x_{i}}=\frac{\partial}{\partial x_{i}}\left(D \varepsilon_{e f f} \frac{\partial \varepsilon}{\partial x_{i}}\right)+\sqrt{2} C_{1 \varepsilon} S_{i j} \varepsilon-C_{2 \varepsilon} \frac{\varepsilon^{2}}{k+\sqrt{\vartheta \varepsilon}}
\end{gathered}
$$

Where $G_{k}$ (Eq. 6) increases the kinetic energy due to mean velocity gradients and is found as follows (Shaheed et al., 2019):

$$
\begin{gathered}
G_{k}=2 \vartheta_{t} S_{i j}^{2} \\
S_{i j}=0.5\left(\frac{\partial u_{j}}{\partial x_{i}}+\frac{\partial u_{i}}{\partial x_{j}}\right) \\
D k_{e f f}=\vartheta+\vartheta_{t} \\
D \varepsilon_{e f f}=\vartheta+\frac{\vartheta_{t}}{\sigma_{\varepsilon}}
\end{gathered}
$$

Where $S_{i j}$ is the strain-rate tensor, $D k_{e f f}$ and $D \varepsilon_{e f f}$ are diffusivity effects for $K$ and $\varepsilon, C_{2 \varepsilon}$ is taken to be 1.9 (constant), $C_{1 \varepsilon}$ is not constant and $\sigma_{\varepsilon}$ is a turbulent Prandtl number assumed to be 1.2. An important feature of the realizable $\mathrm{k}-\varepsilon$ model is that it does not assume $C_{\mu}$ to be constant; it calculates it for the turbulence viscosity (Shaheed et al., 2019).

Wall functions are boundary conditions and in OpenFOAM all of them inherit from the abstract class FvPatchField (Fangqing, 2016). In this paper epsilonWallFunctions, kqRWallFunctions, and nutWallFunctions are used.

\section{PROBLEM SETUP}


The B test Hacker et al., (1996) was modeled in a direct channel and called SC to validate the present study. The length (L), width (b) and height $(\mathrm{H})$ of the model geometry were 3480 , 200 and $500 \mathrm{~mm}$, respectively, and those of the Lock at its beginning ( $\mathrm{x}_{0}, \mathrm{~b}$ and $\mathrm{h}$ - Fig. 2a) were 400, 200, and $400 \mathrm{~mm}$, respectively. In this paper, the fluids' density difference in the SC and research models (Fig. 2b) was $12 \%$. The latter contained a symmetrical $120^{\circ}$ bend in the middle of the obstacle channel. The bend radius from $\mathrm{O}$ to the geometry center (where $\mathrm{z}=0$ ) was considered as $1 \mathrm{~m}$ to ensure the centrifugal force efficacy from center to the channel. In general, the channel geometric dimensions are based on Mahdinia's (2012) paper. Considering the lock length, bend radius and channel angle, studies can be expected to be done in the constant velocity phase. The coordinate system varies along the channel length, the $\mathrm{x}$ direction is always normal to the flow section and point $\mathrm{x}=0$ is somewhere between the lock end and the bend beginning and point $\mathrm{z}=0$ is the center of curvature. Gravity is opposite the $\mathrm{y}$ direction and $\mathrm{y}=0$ is a point at the channel bed. The $120^{\circ}$ bend is connected to the lock on one side and to a Lock-size rectangle on the other. The channel width and its Lock dimensions are equal to those of the validation model. Different bend angles $(\theta)$ are shown dimensionless by dividing in the channel angle $\left(\theta_{t}\right)$.
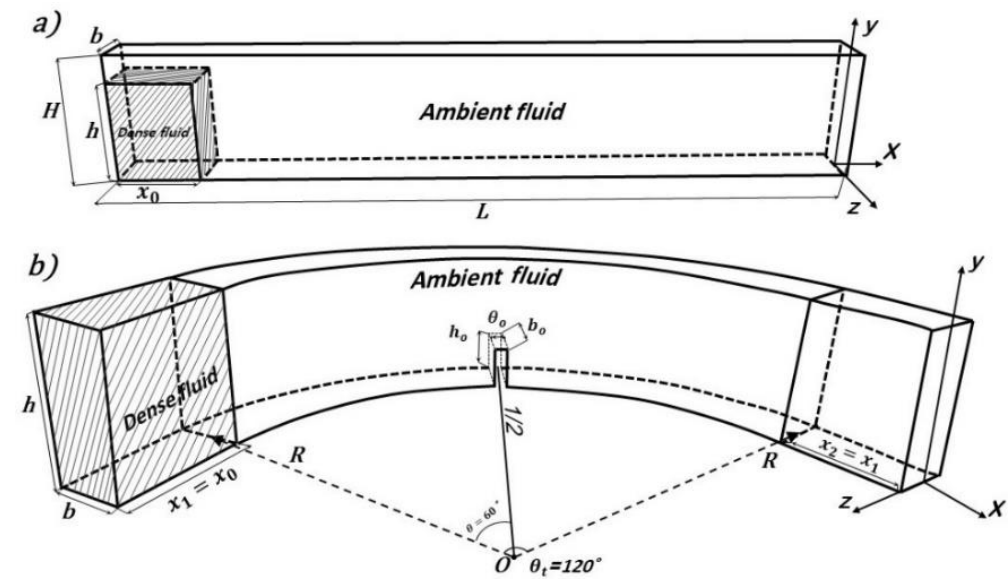

Fig. 2. 3D views of the studied geometries: a) Lab channel (Hacker, 1996) used for validation, b) Curved channel used in present study's models 
In all models, obstacle dimensions are fixed, its height is $0.3 \mathrm{~h}$ (Tokyay and Constantinescu, 2015) and a hypothetical line in the middle of its width makes a $180^{\circ}$ angle with the bend radius; Table (1) summarizes all the geometric parameters. In this paper, BOSD60 is the symbol for bending channel with obstacle in section $60^{\circ}$ and $\mathrm{BC}$ means a no-obstacle curved channel. BOSD30 (obstacle in section $30^{\circ}$ ) has been modeled to study the effects of the obstacle distance from the lock because the bend is symmetrical and its radius is constant along the channel length. Finally, the bending channel with obstacle in section $60^{\circ}$ with increased radius has been modeled with an obstacle at $60^{\circ}$ position (BOSD60IR) and the results have been compared with those of BOSD60. Since the lab modeling of a meandering river with varying-radius curves along the channel length is quite costly, this study has assumed an asymmetric bend made with different-radius circular pieces to study the effects of the obstacle positions on an asymmetric bend by comparing BOSD60IR and BOSD60 models.

The $t / t_{0}$ in Table 1 is the run time in each model wherein the flow front reaches the channel end. During the solution, $\Delta \mathrm{t}$ is a variable (in present models, it varies in a $0.0005-0.009$ ” range) and the Courant maximum is used in the solver's "controlDict" file to ensure its value will not exceed.

Table I. Physical parameters of the present study modeling

\begin{tabular}{ccccccccccccc}
\hline $\begin{array}{c}\text { Symbol } \\
\text { model }\end{array}$ & $\begin{array}{c}\text { Obstacle position } \\
\text { with degree unit }\end{array}$ & $x_{0} / h$ & $\mathrm{~b} / \mathrm{h}$ & $x_{1} / x_{0}$ & $x_{2} / x_{0}$ & $\theta_{o} / \theta_{t}$ & $h_{o} / h$ & $b_{o} / b$ & $\begin{array}{c}\mathrm{R} / \mathrm{h} \\
t / t_{o}\end{array}$ & $\begin{array}{c}\text { Courant } \\
\text { maximum }\end{array}$ \\
\hline SC & - & 1 & 0.512 & - & - & 0.019 & 0.3 & 1 & - & 6.8 & 0.5 \\
BC & - & 1 & 0.512 & 1 & 1 & 0.019 & 0.3 & 1 & 2.5 & 14.2 & 0.3 \\
BOSD30 & 30 & 1 & 0.512 & 1 & 1 & 0.019 & 0.3 & 1 & 2.5 & 17.15 & 0.3 \\
BOSD60 & $\mathbf{6 0}$ & 1 & 0.512 & 1 & 1 & 0.019 & 0.3 & 1 & 2.5 & 16.98 & 0.3 \\
BOSD60IR & 60 & 1 & 0.512 & 1 & 1 & 0.019 & 0.3 & 1 & $\mathbf{3 . 5}$ & 21.6 & 0.3 \\
\hline
\end{tabular}

\section{SOLVER VALIDATION}

The lab channel Hacker et al. (1996) is straight (Fig. 2a) and its dimensions are shown in Table (1) with SC. As stated before in detail, settings in the numerical model are exactly the 
same in both curved and straight channel models. Organized meshes generated in the validation model by the "blockMesh" program, get finer towards the channel bed by a factor of 4 . To ensure the mesh quality, the model was run with 1,2 and 3 million cells in all of which the results, including the data of the flow front position versus time and the general flow shape were equal, but the 3-million cell computational network (Fig. 3) performed better in presenting the mixing of vortices.

Fig. 3 shows the gravity-flow contour lines of the present study's model validated with Hacker et al. (1996) and Mahdinia et al. (2012) lab models under conditions where the flow front position $\left(\mathrm{x}_{\mathrm{f}}\right)$ in the channel lies in a similar place. Results of the present numerical model in early moments differ from those of the lab model; in the latter, compared to the former, lower flow layers move ahead of the upper ones (Simpson, 1972) because of the numerical models' boundary conditions. Although results of the LES turbulence model simulation Ooi et al. (2006) were similar, he attributed it to lab errors when removing the gate in the lab. For more details see Paik et al. (2009).

Solving micro-current vortices by Mahdinia's (2012) LES model cannot be a defect and selecting a RANS turbulence model can be a good option here because of the research objectives and the models' high computational costs. As shown in Fig. (3d), the present model has nicely modeled the flow front collapse near the channel bed. In Mahdinia's (2012) simulation model, the tail of the dense current is at a lower height than its head. It is worth noting that in the lab model, the flow tail vortex is slightly further back along the channel length due, maybe, to the lab gate removal error. In this figure, the bases to compare the tail heights with those of the lab model are points where these heights are the maximum. 
(a)
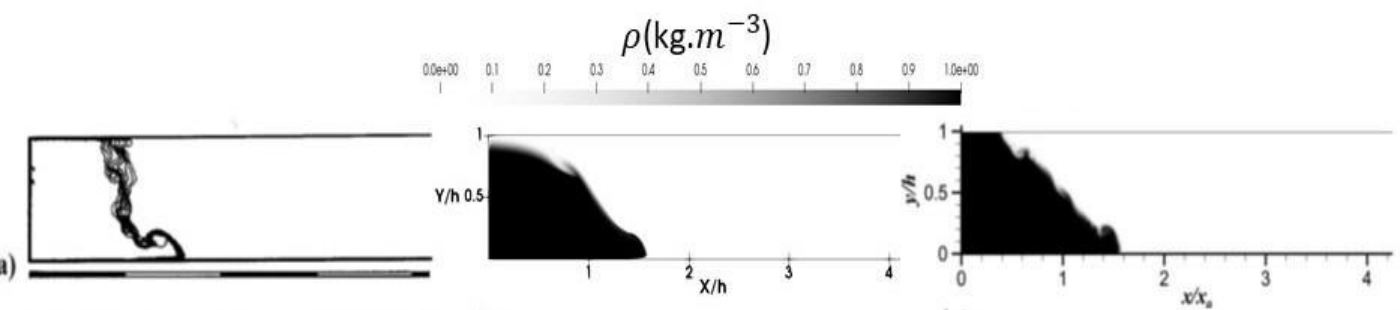

(b)
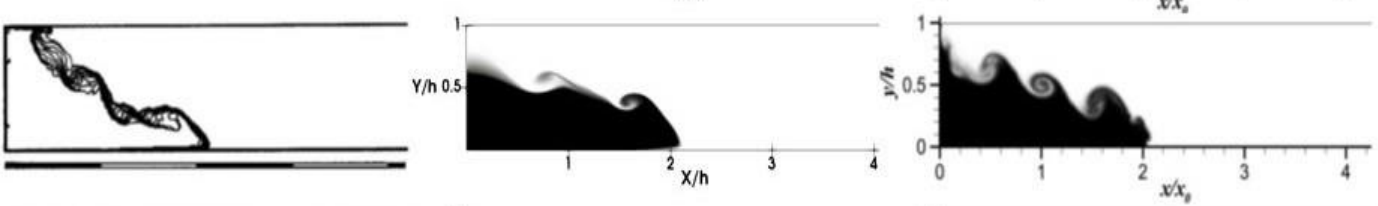

(c)
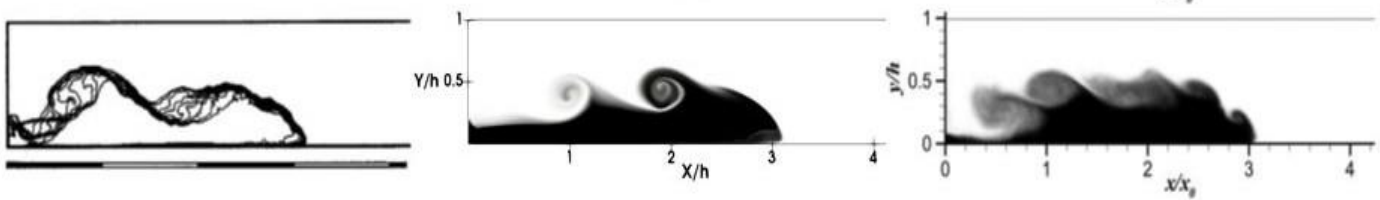

(d)
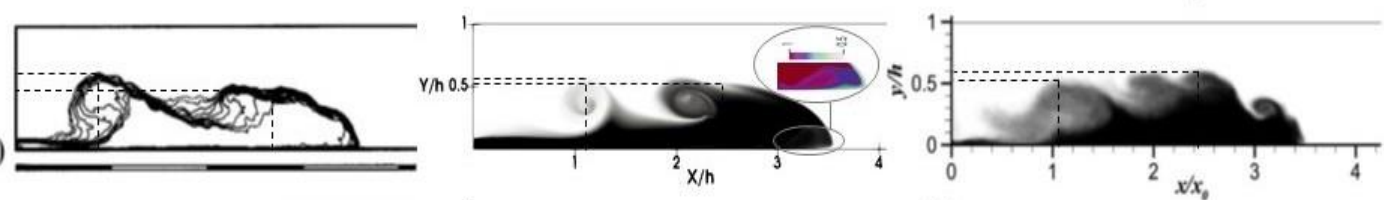

(e)
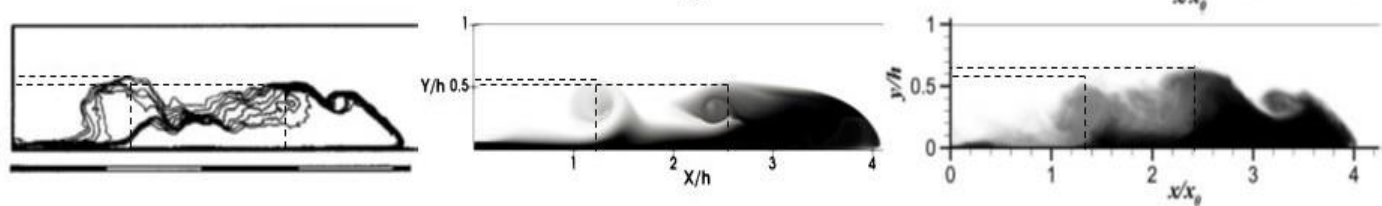

Fig. 3. Contours of the concentration lines for SC model: Hacker's (1996) lab results (left), Mahdinia's (2012) simulation (right) and present study's numerical results (middle) at $\mathrm{t} / \mathrm{t}_{0}$ of a):1.4, b):2.4, c):4.36, d):6 and e):6.26, respectively.

Fig. 4 shows the front position versus time for SC and BC models of this study, Mahdinia et al. (2012) and Hacker et al. (1996) lab model. In curved models, $x_{f}=r \frac{\theta}{\theta_{t}}$ where $x_{f}$ is the front position, $\mathrm{r}$ is bend radius and $\frac{\theta}{\theta_{t}}$ is front position in the curved channel. As mentioned before, the geometric dimensions of the studied channel are based on Mahdinia et al. (2012) with the difference that his is $90^{\circ}$, but that in this study continues up to $120^{\circ}$. Results have shown that the present model data are consistent with those being compared, the flow front moves at a constant speed and the curve gradient in BC model is reduced. 


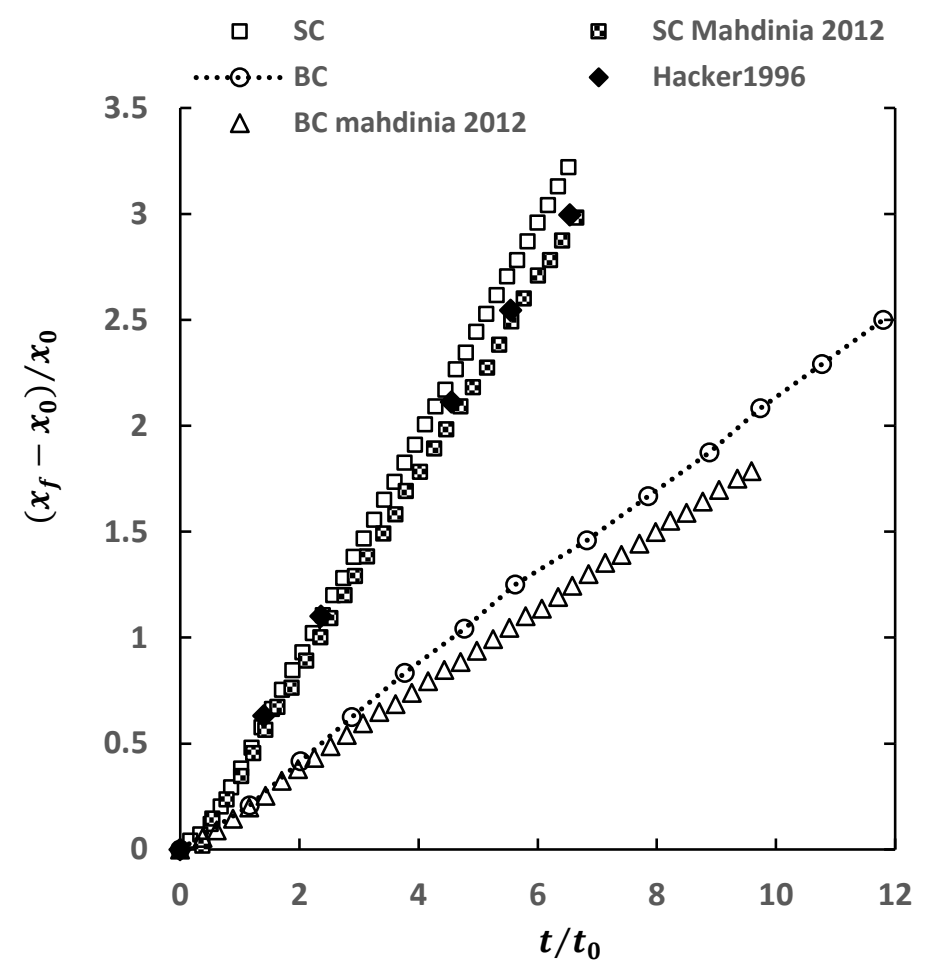

Fig. 4. Front position versus time in validation and research models

\section{GRID SENSITIVITY STUDY}

Since the flow pattern varies in three directions in curved channels causing complicated computational network and increased related costs due to the obstacle presence in the channel, high Reynolds number and 2-phase problem, this research has performed grid sensitivity study for BC, BOSD30, BOSD60 and BOSD60IR models.

Grids have been generated by the blockMesh program that produces organized background grids. Here, the latter are three degrees finer towards the channel bed wall and are completed by the snappyHexMesh program that creates the final computational grid by generating different shape/size cells.

During processing, when the flow front was around the obstacle, the reduced value of $\Delta t$ caused the solution to diverge due, maybe, to the intricate-shape grids around the obstacle. In other words, the snappyHexMesh program generates, for the solver, intricate- and (sometimes) 
incomprehensible-shape cells around the obstacle due to the channel curvature making convergence difficult; this problem will be solved with some changes in the background mesh. All the research models of the present study have been solved by keeping a maximum value of 0.3 for the Courant number. Table (2) lists the specifications of the grid independence runs.

Table II. Grid sensitivity details of the present study

\begin{tabular}{cccc}
\hline Simulation model & Model1 & Model2 & Model3 \\
\cline { 2 - 4 } & $\begin{array}{c}\text { Number of } \\
\text { cell in } \\
\text { mesh } \\
\text { (millions) }\end{array}$ & $\begin{array}{c}\text { Number of } \\
\text { cell in } \\
\text { mesh } \\
\text { (millions) }\end{array}$ & $\begin{array}{c}\text { Number of } \\
\text { cell in } \\
\text { mesh } \\
\text { (millions) }\end{array}$ \\
\hline BC & 1.43 & 3.36 & 4.22 \\
BOSD60 & 1.42 & 3 & 3.89 \\
BOSD60IR & 1.39 & 3.01 & 3.96 \\
BOSD30 & 1.42 & 3.35 & 5.8 \\
\hline
\end{tabular}

The front position versus time was the first parameter studied and the results were almost equal in all different-volume models, i.e. closer results even in smaller grid volumes. Next, such parameters as pressure, concentration and velocity in different directions were investigated where the highest difference in results was observed in concentration contours, especially in the front position. As shown in Fig. 5, results are, in general, quite close with no major difference among the three computational grids. However, since the highest number of cells in each model had closer results than the two models with the lowest number of cells, the highest number of cells in each model was selected as the final grid. It is worth mentioning that Fig. 5 has used different-color spectrum and different-angle models for a better comparison. 

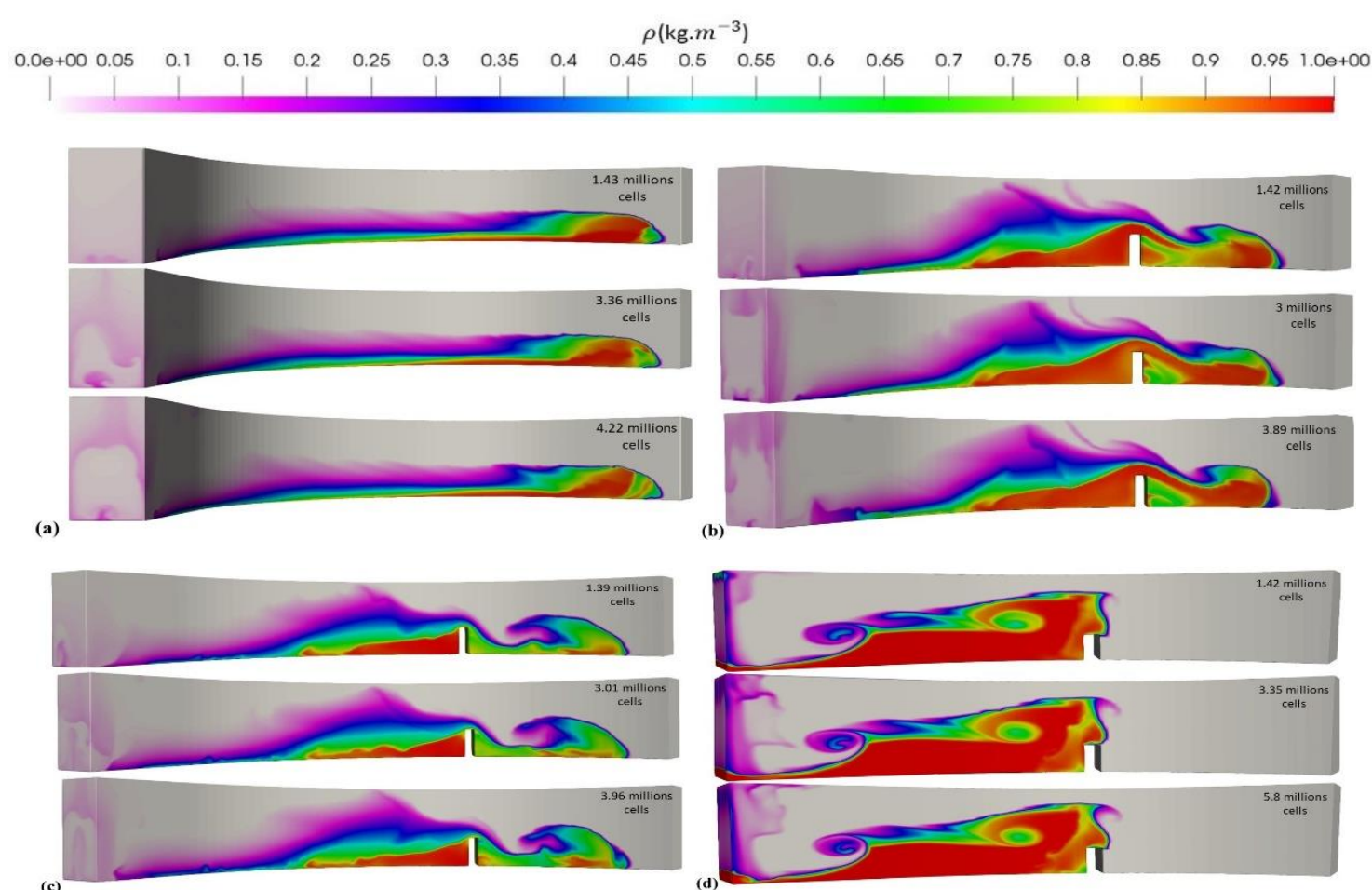

Fig. 5. Concentration isosurfaces from the inner bank view: a) $B C$ model at $t / t_{0}=13.7$ front position near the channel end, b) BOSD60 model at $\mathrm{t} / \mathrm{t}_{0}=11.15$, front position at $3 / 4$ of the channel length, c) BOSD60IR model at $t / t_{0}=14.58$, front position at $3 / 4$ of the channel length, d) BOSD30 model at $t / t_{0}=4.46$, front position at $1 / 4$ of the channel length

In the present study, the sensitivity data of the velocity along the channel width, especially near the bed, are very close, but since its main objective is the analysis of the secondary flow, its results are shown in Fig. 6; results in the longitudinal velocity and pressure parameters are almost the same. 

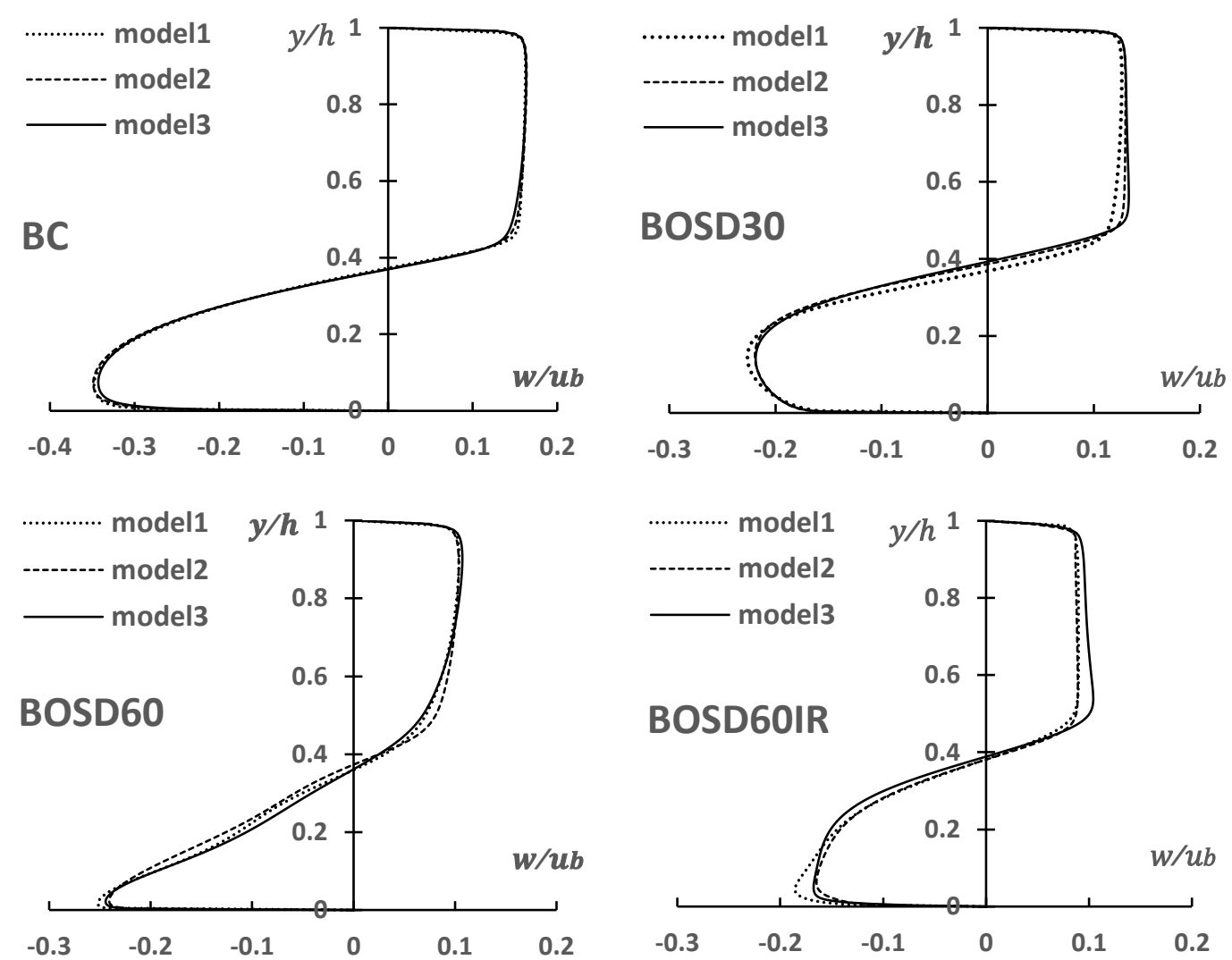

Fig. 6. Comparison of the transverse velocity data in grid sensitivity studies at $\mathrm{z}=0$ position

Fig. 6 data are related the front position at the channel end and $z=0$ point is at $3 / 4$ of the channel length. As shown, results are quite close, and selecting "the maximum number of cells" as the final grid in each model is acceptable. In general, obstacles reduce the secondary flow, but comparison of the BOSD30 and BOSD60 models in this figure shows that when the obstacle nears the lock, the secondary flow in the main body increases after the obstacle $(3 / 4$ length position) while in the channel bed, the secondary flow is more in the BOSD60 model. It is worth noting that under different flow-body positions after the obstacle results are similar (but not given). Since relations are none for the secondary flow in Lock-exchange currents in curved channels, precise reasoning is not possible, but high current energy and longitudinal velocity at initial moments can be the reasons for it. However, these results are in line with the finding of $\mathrm{Wu}$ and Ouyang (2020) who investigated Flow morphology of gravity currents interacting with immersed obstacles in a direct channel. They showed the time history of the 
normalized potential energy, kinetic energy, and dissipation rate of gravity currents before, during, and after the impact. They identified various stages of propagation, including the impact, transient, and quasi-steady stages.

\section{DISCUSSION AND RESULTS}

This section is to study the results of the no-obstacle curved channel model (BC) and that with obstacle at $60^{\circ}$ position (BOSD60), and compare the results to check the obstacle effects. Comparing BOSD60 and BOSD60IR will enable the studying of the increased radius effects (with obstacle in channel), and comparing BOSD30 and BOSD60 (when obstacle nears the lock) will enable the studying of the obstacle displacement effects on the flow.

\section{Flow Front Position}

In curved channels, $x_{f}=r \frac{\theta}{\theta_{t}}$ where $x_{f}$ is the front position, $\mathrm{r}$ is bend radius and $\frac{\theta}{\theta_{t}}$ is front position in the curved channel. As shown in Fig. 7, the front in the BC model moves at a constant speed and the line gradient is constant. In BOSD60, BOSD30 and BOSD60IR models, the gradient is equal to that in the $\mathrm{BC}$ model in the pre- and post-obstacle modes (where the front is not affected by the obstacle). Under the obstacle effect, the gradient is reduced and reaches zero $(\mathrm{m}=0)$ where the nose falls ahead in the outer bank. This means sometimes, while the flow maintains its dynamicity, the front does not fall ahead; rather, it is the nose that does so. 


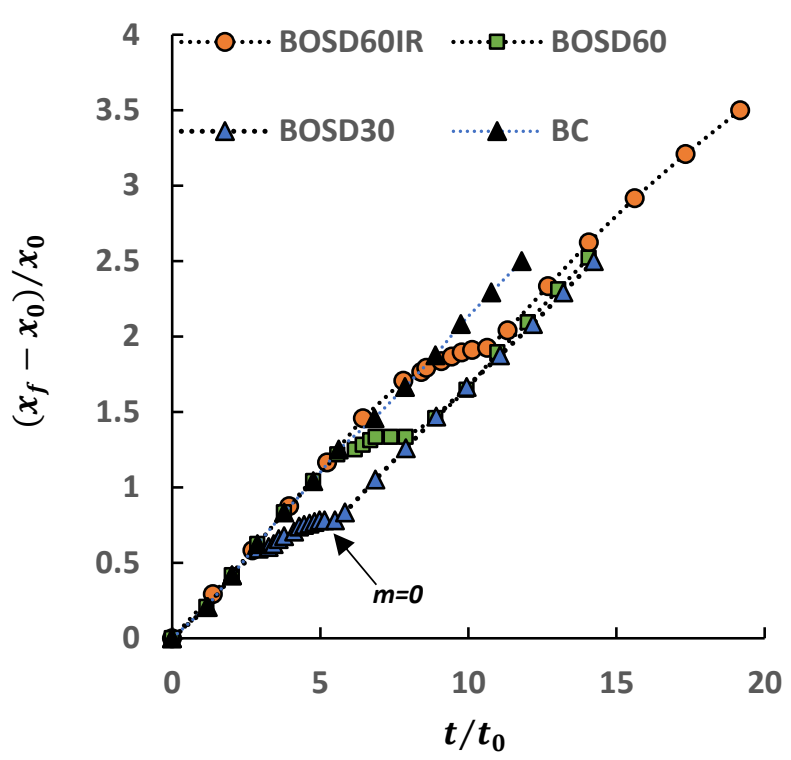

Fig. 7. Front position versus time in research models

Discussed in detail by Mahdinia et al. (2012), in the BC model, the nose falls ahead in the inner bank in the first half of the channel and then tends to do so in the outer bank after the obstacle if there is one. Since this is the case in BOSD30 model, it can be concluded that the mere obstacle causes the nose to fall ahead in the outer bank in some part of its length and the obstacle position at the bend is irrelevant; time intervals when the gradient is zero are different in each model. These results are also in a good agreement with $\mathrm{Wu}$ and Ouyang (2020) numerical research that investigated flow morphology in bottom-propagating gravity currents over immersed obstacles in a direct channel. Leong et al. (2006) also showed the similar results in a direct channel and mentioned that the velocity of the advancing front fluctuated as the current moved along.

\section{Obstacle in the Channel Bend}

The lock-released gravity current is a time-dependent deformable "obstacle," whose shape interacts with the waves it produces in the ambient and analysis and investigation of the field 
of time-dependent flow is obviously a difficult task (Ungarish, 2009). This section is aimed to discuss the curved-channel obstacle effects on the contours of the longitudinal and transverse velocities and the concentration. To this end, different channel-width sections of the BOSD60 model at different obstacle positions were compared with similar-position results of the BC model when the flow front was in the 11/12 channel position. Sections at 5/6, 4/6, 3.25/6 and 1.75/6 channel positions were considered to compare the flow head, tail, post-obstacle tail and pre-obstacle tail structures, respectively.

In a direct channel, entrainment was shown to increase downstream of the obstacle when the obstacle was present as shown by Wilson et al (2017). In the same way, our present results show this flow feature in a bend. Fig. 8 shows contours of the concentration in the channel cross section; in $8 \mathrm{a}(5 / 6$ position), the head height has found its shape, is almost equal in both models, is not affected by the obstacle, concentration is highly reduced due to the obstacle and is almost maximum near the channel bed on both inner and outer banks. In $8 \mathrm{~b}$ (4/6 position), obstacles reduce the tail height and maximum concentration is in the inner bank. In the $\mathrm{BC}$ model, concentration increases in the tail in the outer bank and reaches its maximum at the channel bed by moving away from the flow front; this maximum is usually in the inner bank if there is an obstacle in different positions. The post-obstacle tail height is almost balanced across the channel due, maybe, to the low flow velocity (8c). Being almost equal across the channel, the pre-obstacle tail height is maximized in the outer bank as it approaches the lock (8d).

A similar conclusion was reached by Wilson et al. (2018) who showed that in a turbidity currents in a direct channel, interacting with an obstacle, the obstacle reduce the maximum velocity and turbulence intensity downstream of it.

Fig. 9 shows contours of the longitudinal velocity across the channel. The flow front is at channel position 11/12 and cross-section positions are as in Fig. 8. The longitudinal velocity 
direction is negative at the flow head, but changes by moving away from the head to the tail. An obstacle causes this to happen sooner; in 9a, the maximum longitudinal velocity at the flow head occurs in the outer bank nearly around $y / h=0.3$. In $9 \mathrm{~b}$, the velocity decrease is quite clear in the tail reaching its maximum near the channel bed. In 9c, the post-obstacle longitudinal velocity distribution is irregular due, maybe, to the vortices and flow turbulence. In 9d, the preobstacle longitudinal velocity direction is negative in the channel bed due, clearly, to a direction change. As shown, the longitudinal velocity is maximized in the inner bank with a quite opposite flow pattern in the channel upper half. In the BC model, the longitudinal velocity is maximized before the vertex in the outer bank and is balanced in the channel width after the vertex.

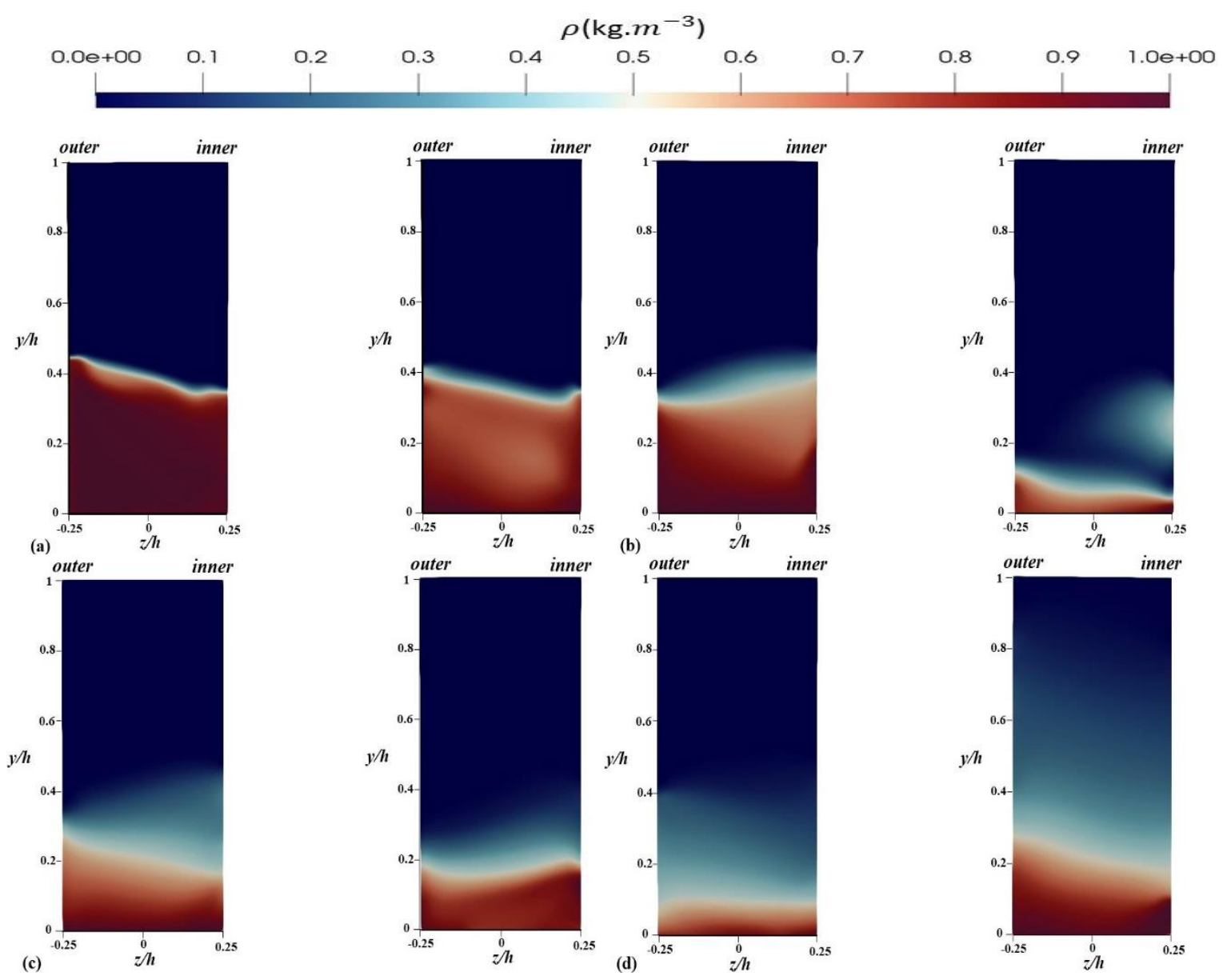

Fig. 8. Concentration contours: cross sections at a)5/6, b)4/6, c)3.25/6 and d)1.75/6 from the channel. BC model at $\mathrm{t} / \mathrm{t}_{0}=10.77$ and flow front at $11 / 12$ position from the channel (left), BOSD60 model at $\mathrm{t} / \mathrm{t}_{0}=13$ and flow front at $11 / 12$ position from the channel (right) 

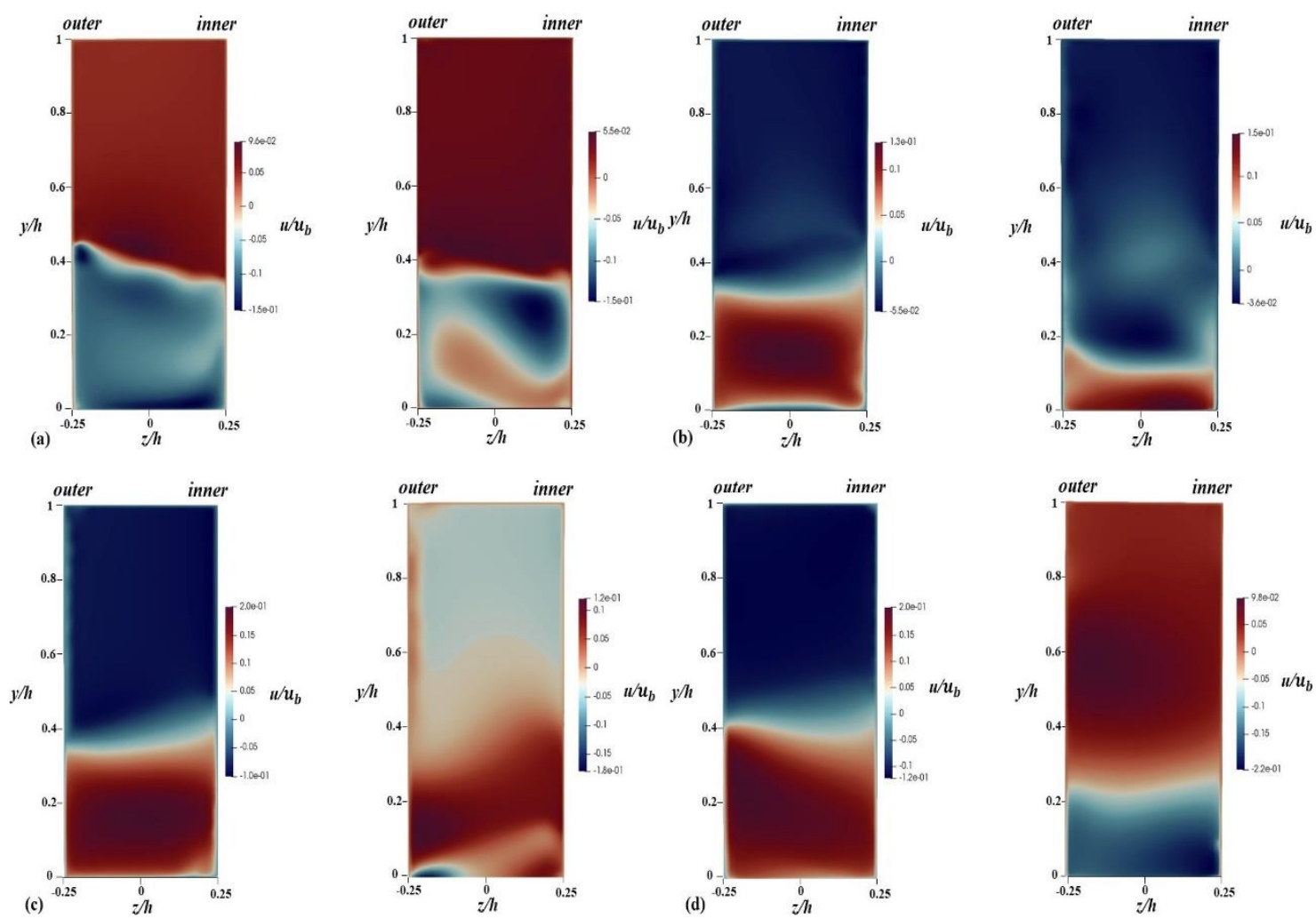

Fig. 9. Longitudinal velocity: cross sections at a)5/6, b)4/6, c)3.25/6 and d)1.75/6 from the channel. BC model at $\mathrm{t} / \mathrm{t}_{0}=10.77$ and flow front at $11 / 12$ position from the channel (left), BOSD60 model at $\mathrm{t} / \mathrm{t}_{0}=13$ and flow front at $11 / 12$ position from the channel (right)

Comparison of Figs 8 and 9 shows that in the BC model, the concentration decreases in the channel wherever the longitudinal velocity increases, but this concentration-longitudinal velocity coordination does not exist around and before an obstacle.

Fig. 10 shows contours of the transverse velocity; like before, the flow front is at channel position $11 / 12$ and a further section can be seen in $10 \mathrm{~d}$ at $2.75 / 6$ which is a pre-obstacle position. In the BC model, the secondary flow pattern is from the inner to the outer bank near the bed, from the outer to the inner bank near the interface and irregular near the tail. Since these results conform to those of Mahdinia et al. (2012), it can be concluded, in general, that obstacles reduce the transverse velocity at different positions with the highest reduction around, especially before, the obstacle (Fig. 10d). According to 10a and 10b, both in post-obstacle positions, the secondary flow direction is similar to that in the $\mathrm{BC}$ model with the difference 
that it decreases in the outer bank and Increases in the inner one. In 10c and 10d, the flow has a different pattern near the obstacle due to vortices and flow turbulence; in the channel bed, the secondary flow direction is from the outer bank to the inner, changes above it and again from the outer to the inner bank in the channel upper half. Near the lock, the direction is totally reverse; from outer to inner near the channel bed, and from inner to outer near the interface.
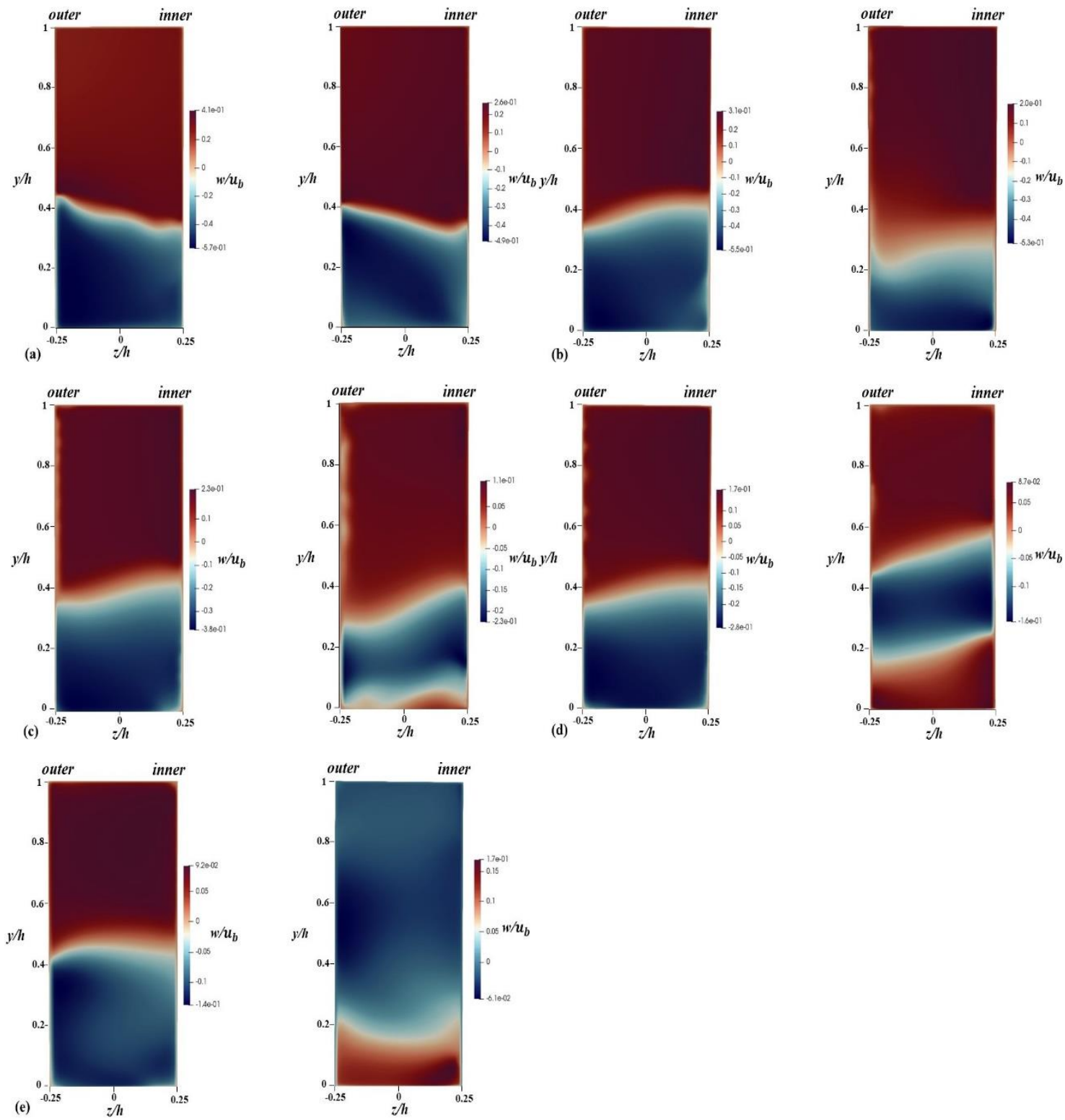

Fig. 10. Transverse velocity: cross sections at a)5/6, b)4/6, c)3.25/6, d)2.75/6 and e) $1.75 / 6$ from the channel. BC model at $\mathrm{t} / \mathrm{t}_{0}=10.77$ and flow front at $11 / 12$ position from the channel (left), BOSD60 model at $\mathrm{t} / \mathrm{t}_{0}=13$ and flow front at 11/12 position from the channel (right) 


\section{Obstacle effects when channel radius increases}

As mentioned before, with an obstacle, an increase in the radius will not change the gradient of the flow front motion graph. Mahdinia et al. (2012) had already shown that in a no-obstacle channel, a change in the radius would not change the front position versus time graph gradient and concluded that the secondary flow would decrease with an increase in the radius. Despite the presence of an obstacle, the present study too has had similar flow pattern results to be described in the following parts.

Figure 11 shows the BOSD60IR model contours in sections 5/6 and 4/6 when the flow front is at position 11/12 to be compared with those of the BOSD60 model. As shown, an increase in the bend radius in the former reduces the centrifugal force and, hence, the velocity compared to the latter. With this increase, the channel-across flow components tend to increase in the banks as well as in other positions. Fig. 11a shows that the heights of flow tail and head have decreased.

\section{Pre-vertex obstacle effects}

According to Mahdinia et al. (2012), the initial-moment maximum longitudinal flow velocity is observed in the inner bank where the flow nose is further ahead in the first half of the channel. Since these results have also been observed in the present study, the obstacle effects, in the BOSD30 model, have also been studied in this half the results of which are shown in Fig. 12. 

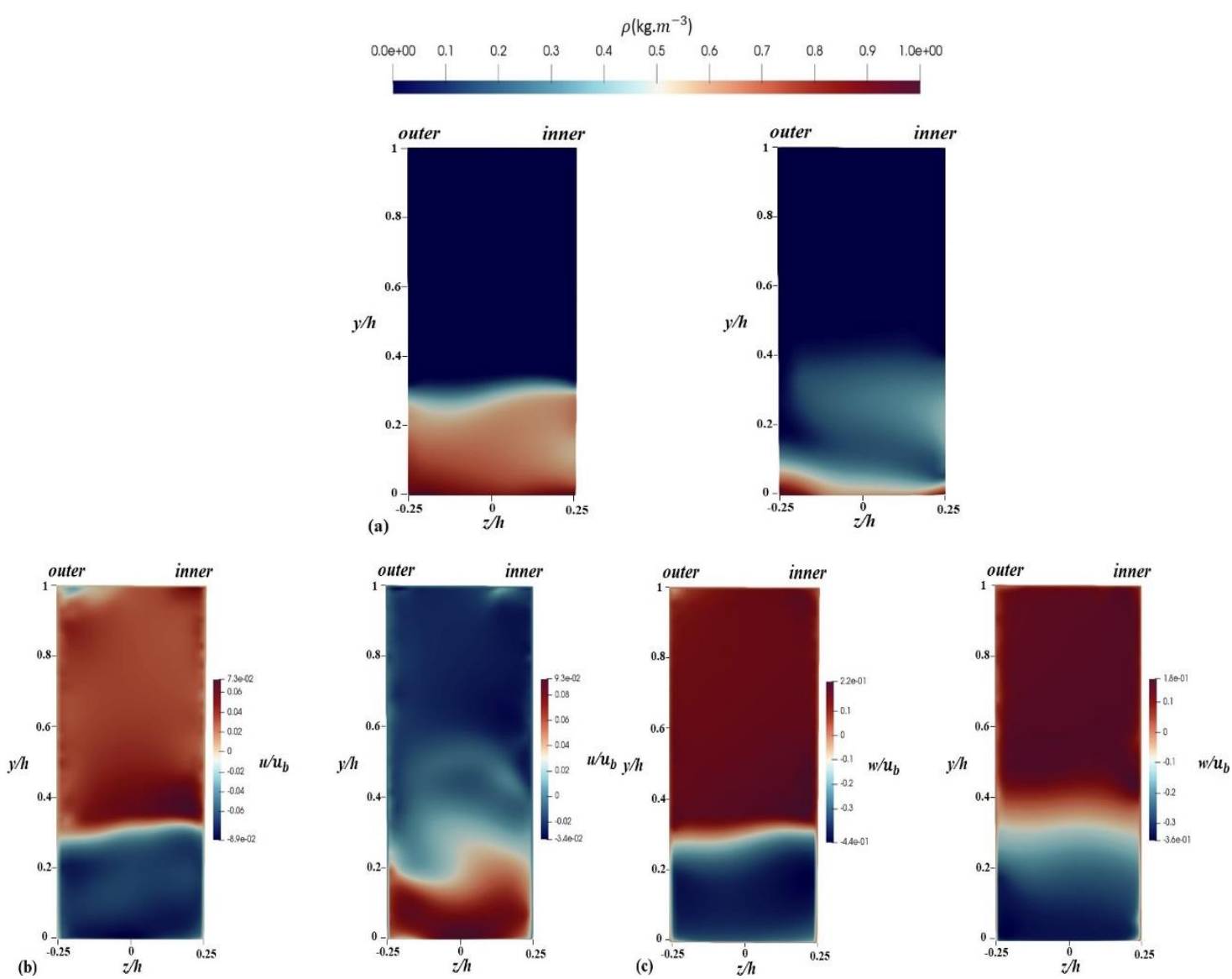

Fig. 11. BOSD60IR model results at $\mathrm{t} / \mathrm{t}_{0}=17.32$ with the flow front at $11 / 12$ position from the channel: section at position 5/6 (left) and position 4/6 (right); a)the concentration, b)longitudinal velocity and c)transverse velocity contours

As mentioned in Fig. 6, in comparing BOSD30 and BOSD60 models, if the flow front is in a similar post-obstacle channel position (e.g., at the end), the secondary flow in the main body is more in BOSD30, but both have similar flow patterns. Now, in Fig. 12, the BOSD30 model results are compared with those of the second part where their post-obstacle flow front positions are $50^{\circ}$. Contours are given at sections 3.5/6 (a flow-head position) and 1.75/6 (a post-obstacle flow-tail position) where the flow front is at position 4/6. 

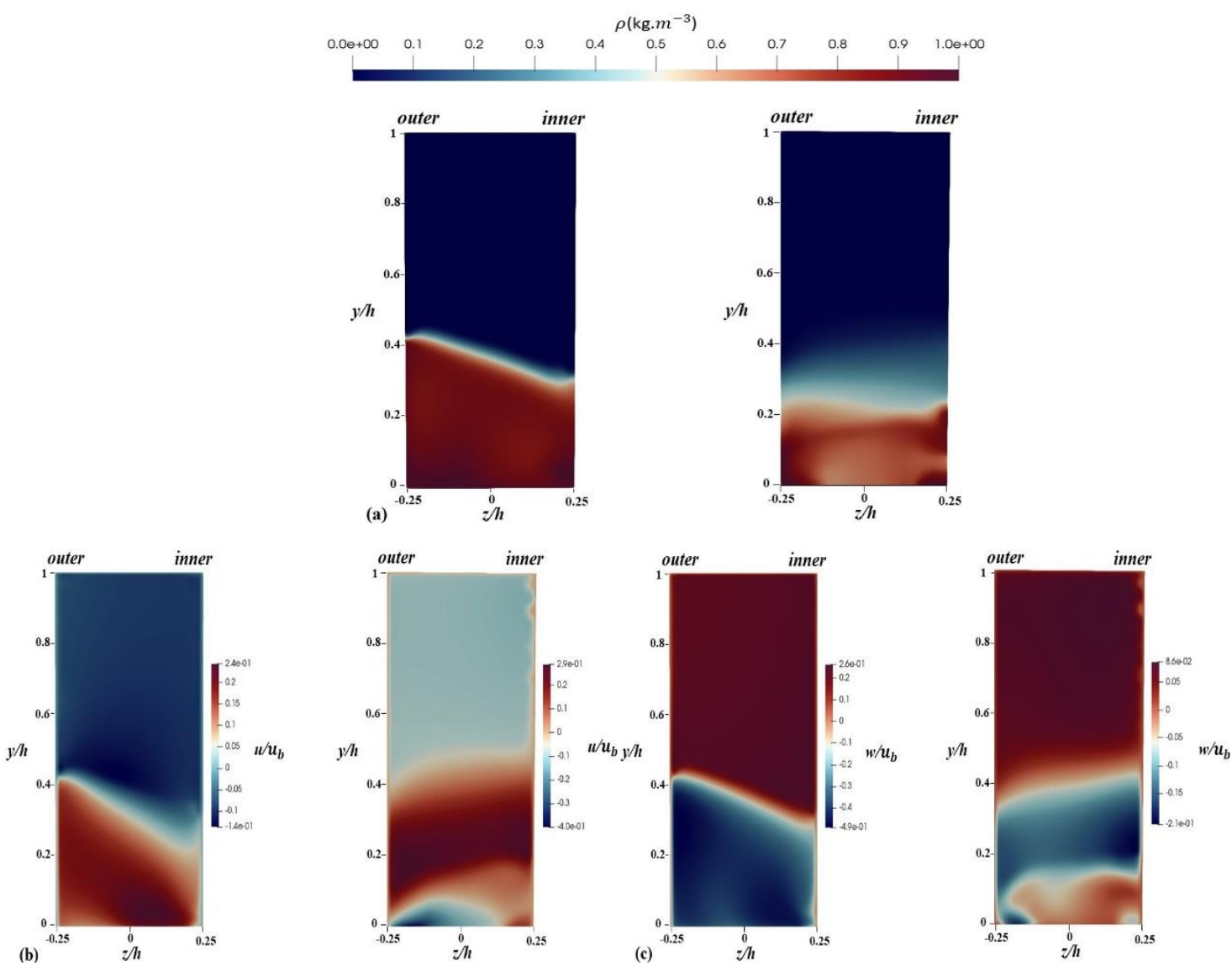

Fig. 12. BOSD30 model results at $\mathrm{t} / \mathrm{t}_{0}=9.94$ with the flow front at $4 / 6$ position from the channel: section at position 3.5/6 (left) and position 1.75/6 (right); a)the concentration, b)longitudinal velocity and c)transverse velocity contours

At 3.5/6, a flow head position in BOSD30 model, concentration tends to increase in the banks compared to similar position in BOSD60 model. A point worth noting is that the longitudinal velocity highly increases with a positive direction in the flow head. According to the observations (pictures not given here), the flow has a positive direction throughout the head except for the nose where the direction is negative near the channel bed. BOSD30 model results show that the longitudinal velocity decreases at the flow head where concentration has increased. Observations (Fig. 12c) show that the secondary flow amount/pattern does not change much compared to the BOSD60 model. 


\section{CONCLUSIONS}

This paper has investigated the effects of the obstacle presence/position in a gravity-flow in a symmetrical curved channel. Settings presented for the numerical model are for good validation results. The mesh sensitivity analyses results show that the transverse velocity parameter is less sensitive to concentration than to the computational network. At the channel beginning, the front moves at a constant speed, and the nose is farther in the inner bank. After hitting the obstacle, its headway speed is reduced, but in post-obstacle conditions, while maintaining its dynamism, the flow front does not move along the channel for a while when its nose gets ahead in the outer bank after which it continues moving at a constant speed.

With an obstacle, the flow-head density is reduced, but the head height does not change. The density is maximized in most channel positions in the inner bank, but in the no-obstacle case, the density increases in the outer bank, especially in the tail. As the radius increases, the head and tail heights of the flow are reduced and the density tends towards the banks. With an obstacle, the longitudinal velocity is highly reduced in the tail and has a maximum value in the channel bed; in the outer bank, it is maximized around the obstacle and in the inner bank it does so far from it.

In general, obstacles reduce the transverse velocity along the channel, but cause different secondary flow patterns at different positions. The highest reduction is observed around, especially before the obstacle. Results show that displacing obstacles enables the secondary flow and its $\min / \max$ values to be controlled and managed along the bend. According to the results of the present study, when the flow front is at the channel end and the obstacle is near the lock, there will be more secondary flow in the lower half of the channel (where the flow direction is toward the outer bank) and its maximum value occurs in the outer bank, but when 
it is at a certain distance from the obstacle, the secondary flow pattern/amount does not change much.

\section{DATA AVAILABILITY STATEMENTS}

The data that supports the findings of this study are available within the article.

\section{ACKNOWLEDGMENTS}

The authors declare that they have no conflict of interest and this research received no specific grant from any funding agency in the public, commercial, or not-for-profit sectors.

\section{REFERENCES}

Abad, J. D., and Garcia, M. H., "Experiments in a high-amplitude Kinoshita meandering channel: 1. Implications of bend orientation on mean and turbulent flow structure," Water Resources Research. 45(2), W02401 (2009a).

Abad, J. D., and Garcia, M. H., "Experiments in a high-amplitude Kinoshita meandering channel: 2. Implications of bend orientation on bed morphodynamics,” Water Resources Research. 45(2), W02402 (2009b).

Balasubramanian, S., and Zhong, O., "Entrainment and mixing in lock-exchange gravity currents using simultaneous velocity-density measurements", Physics of Fluids 30, 056601 (2018).

Brice, J. C., "Evolution of meander loops.” Geological Society of America Bulletin. 85(4), 581-586 (1974).

Ezz, H., Cantelli, A., and Imran, J., "Experimental modeling of depositional turbidity currents in a sinuous submarine channel," Sedimentary Geology. 290, 175-187 (2013).

Farshi, F., Kabiri-Samani, A., Chamani, M. R., and Atoof, H., "Evaluation of the secondary current parameter and depth-averaged velocity in curved compound open channels," Journal of Hydraulic Engineering. 144(9), 04018059 (2018).

Fangqing L, "A Thorough Description Of How Wall Functions Are Implemented In OpenFOAM", In Proceedings of CFD with OpenSource Software, Edited by Nilsson. H., (2016).

Firoozabadi, B., Afshin, H., and Aram, E., "Three-dimensional modeling of density current in a straight channel," Journal of Hydraulic Engineering. 135(5), 393-402 (2009). 
Hacker, J., Linden, P. F., and Dalziel, S. B., "Mixing in lock-release gravity currents,” Dynamics of Atmospheres and Oceans. 24(1-4), 183-195 (1996).

Islam, M. A., and Imran, J., "Experimental modeling of gravity underflow in a sinuous submerged channel,” Journal of Geophysical Research: Oceans. 113(C7) (2008).

Kassem, A., and Imran, J., "Three-dimensional modeling of density current. II. Flow in sinuous confined and uncontined channels,” Journal of Hydraulic Research. 42(6), 591-602 (2004).

Lam, W. K., Chan, L., Hasini, H., and Ooi, A., "Direct numerical simulation of two-dimensional stratified gravity current flow with varying stratification and aspect ratio," 21st Australasian Fluid Mechanics Conference, AFMC 2018., Australasian Fluid Mechanics Society (2018).

Leong, K.K., Mok, K.M. \& Yeh, H. "Fluctuation of the front propagation speed of developed gravity current", Journal of Hydrodynamics. 18, 343-347 (2006).

Lombardi, V., Adduce, C., Sciortino, G., and La Rocca, M., “Gravity currents flowing upslope: Laboratory experiments and shallow-water simulations," Physics of Fluids. 27(1), 016602 (2015).

Longo, S., Ungarish, M., Di Federico, V., Chiapponi, L., and Maranzoni, A., "The propagation of gravity currents in a circular cross-section channel: experiments and theory,” Journal of Fluid Mechanics. 764, 513-537 (2015).

Magdaleno, F., and Fernández-Yuste, J. A., "Meander dynamics in a changing river corridor," Geomorphology. 130(3-4), 197-207 (2011).

Mahdinia, M., Firoozabadi, B., Farshchi, M., Varnamkhasti, A. G., and Afshin, H., "Large eddy simulation of Lock-Exchange flow in a curved channel," Journal of Hydraulic Engineering. 138(1), 57-70 (2012).

Meiburg, E., Radhakrishnan, S., and Nasr-Azadani, M., "Modeling gravity and turbidity currents: computational approaches and challenges,” Applied Mechanics Reviews. 67(4), (2015).

Ooi, S. K., Constantinescu, G., and Weber, L. J., "2D large-eddy simulation of lock-exchange gravity current flows at high Grashof numbers,” Journal of Hydraulic Engineering, 133(9). 1037-1047 (2007).

Ottolenghi, L., Adduce, C., Inghilesi, R., Armenio, V., and Roman, F., "Entrainment and mixing in unsteady gravity currents,” Journal of Hydraulic Research. 54(5), 541-557 (2016). 
Ottolenghi, L., Adduce, C., Inghilesi, R., Roman, F., and Armenio, V., "Mixing in lock-release gravity currents propagating up a slope,” Physics of Fluids. 28(5), 056604 (2016).

Paik, J., Eghbalzadeh, A., and Sotiropoulos, F., “Three-Dimensional unsteady RANS modeling of discontinuous gravity currents in rectangular domains," Journal of Hydraulic Engineering. 135(6), 505-521 (2009).

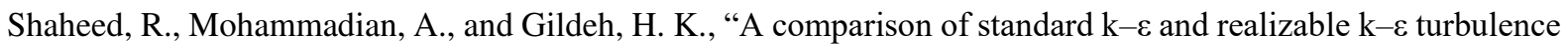
models in curved and confluent channels," Environmental Fluid Mechanics. 19(2), 543-568 (2019).

Simpson, J. E., "Gravity Currents: in the Environment and the Laboratory," Cambridge university press, Cambridge, UK (1999).

Sumner, E. J., Peakall, J., Dorrell, R. M., Parsons, D. R., Darby, S. E., Wynn, R. B., ... and White, D., "Driven around the bend: Spatial evolution and controls on the orientation of helical bend flow in a natural submarine gravity current,” Journal of Geophysical Research: Oceans. 119(2), 898-913 (2014).

Tokyay, T., Constantinescu, G., and Meiburg, E., “Tail structure and bed friction velocity distribution of gravity currents propagating over an array of obstacles," Journal of fluid mechanics. 694, 252-291 (2012).

Tokyay, T., and Constantinescu, G., "The effects of a submerged non-erodible triangular obstacle on bottom propagating gravity currents," Physics of fluids. 27(5), 056601 (2015).

Ungarish, M., “An introduction to gravity currents and intrusions," Israel Institute of Technology Haifa, CRC press (2009).

Wilson R., Friedrich H., and Stevens, C., "Turbulent entrainment in sediment-laden flows interacting with an obstacle", Physics of Fluids 29, 036603 (2017).

Wilson R., Friedrich H., and Stevens, C., "Flow structure of unconfined turbidity currents interacting with an obstacle”, Environmental Fluid Mechanics, volume 18, 1571-1594(2018).

Wu, Ch and Ouyang, H., "Flow morphology in bottom-propagating gravity currents over immersed obstacles", AIP Advances. 10, 115103 (2020).

Xie P. and Chu V., "OpenFoam simulation of gravity-current head impact on a structure on the seafloor", Eproceedings of the 38thIAHR World Congress, 2019. 
Zhang, S., Jiang, B., Law, A. W. K., \& Zhao, B. “Large eddy simulations of 45 inclined dense jets”, Environmental Fluid Mechanics,16(1), 101-121 (2016).

Zhao, L., Ouillon, R., Vowinckel, B., Meiburg, E., Kneller, B., and He, Z., “Transition of a hyperpycnal flow into a saline turbidity current due to differential diffusivities", Geophysical Research Letters. 45(21), 11-875 (2018).

Zhou, J., and Venayagamoorthy, S., "How does three-dimensional canopy geometry affect the front propagation of a gravity current?", Physics of Fluids 32, 096605 (2020).

"Guide, turbulence, RANS, realizable-k-epsilon.” Accessed December 25, (2020). https://www.openfoam.com/documentation/guides/latest/doc/guide-turbulence-ras-realizable-k-epsilon.html 